\title{
A Coarse-Grained Model for the Mechanical Behavior of Graphene Oxide
}

Zhaoxu Meng ${ }^{1+}$, Rafael A. Soler-Crespo ${ }^{2 \dagger}$, Wenjie Xia ${ }^{1}$, Wei Gao ${ }^{3}$, Luis Ruiz ${ }^{4}$, Horacio D. Espinosa $^{2,3 *}$ and Sinan Keten ${ }^{1,2 *}$

${ }^{1}$ Department of Civil and Environmental Engineering, Northwestern University, 2145 Sheridan Road, Evanston, IL 60208-3111, United States

${ }^{2}$ Theoretical and Applied Mechanics Program, Northwestern University, 2145 Sheridan Road, Evanston, IL 60208-3111, United States

${ }^{3}$ Department of Mechanical Engineering, Northwestern University, 2145 Sheridan Road, Evanston, IL 60208-3111, United States

${ }^{4}$ Chemical Sciences Division, Lawrence Berkeley National Laboratory, University of California, Berkeley, CA 94720, United States

${ }^{\dagger}$ These authors contributed equally to this work

* Corresponding authors: s-keten@,northwestern.edu, espinosa@northwestern.edu 


\begin{abstract}
Graphene oxide (GO) shows promise as a nanocomposite building block due to its exceptional mechanical properties. While atomistic simulations have become central to investigating its mechanical properties, the method remains prohibitively expensive for large deformations and mesoscale failure mechanisms. To overcome this, we establish a coarse-grained (CG) model that captures key mechanical and interfacial properties, and the non-homogeneous effect of oxidation in GO sheets. The CG model consists of three types of CG beads, representing groups of pristine $s p^{2}$ carbon atoms, and hydroxyl and epoxide functionalized regions. The CG force field is parameterized based on density functional-based tight binding simulations on three extreme cases. It accurately quantifies deterioration of tensile modulus and strength at the expense of improving interlayer adhesion with increasing oxidation of varying chemical compositions. We demonstrate the applicability of the model to study mesoscale phenomena by reproducing different force vs. indentation curves in silico, corroborating recent experimental observations on how chemistry near contact point influences properties. Finally, we apply the model to measure the fracture toughness of pristine graphene and GO. The critical stress intensity factor $\left(K_{c}\right)$ of graphene is found to be the highest, and epoxide-rich GO also possess higher $K_{c}$ compared to hydroxyl-rich GO.
\end{abstract}




\section{Introduction}

Graphene has emerged as a promising building block that can be used in nanocomposites and nanoelectronic devices to gain advances in mechanical and electronical performance [1-6]. However, it has been shown through experiments and theory that load transfer between stacked graphene sheets and between graphene sheets and polymer matrices is poor as it employs weak van der Waals forces [7-10]. Graphene oxide (GO), a derivative of graphene, contains oxygenrich functional groups capable of hydrogen bonding, which addresses this issue [11-13], and allows dispersion of graphitic sheets in many solvents and polymer matrices in nanocomposites [14-16]. Although the existence of functional groups in GO deteriorates its in-plane mechanical properties, i.e. Young's modulus and intrinsic strength, compared to pure graphene, significant enhancement in its capability of damage tolerance and the interfacial interactions possible between GO and matrix materials makes GO favorable in many applications [17-23]. GO-based fillers have been used in polymer nanocomposites [24-26], and recent studies have emphasized the importance of surface functionalization for a number of reasons. For example, recent studies have shown that increasing the adhesion energy between graphitic sheets and reducing the tensile modulus of each sheet, as in the case of oxidation, allows multi-layer graphitic systems to attain high strength with little sheet overlap [27]. In addition, nanoconfinement of polymers in nanocomposites systems becomes effective only when the polymer-graphitic filler interactions are highly attractive, leading to matrix stiffening in nanocomposites [28, 29]. Moreover, it has been reported that thermal stability [30-33] and electrical properties [34-37] of polymers could be greatly improved by incorporation of GO nanosheets.

Atomistic modeling has been extensively utilized to investigate and predict the mechanical properties of GO. Most prior investigations on GO modeling were carried out using all-atomistic 
(AA) simulations or first principles calculations, which provide valuable insights into molecular mechanisms governing constitutive behavior while retaining critical chemical details. It is expected that the surface functional groups have considerable effects on the mechanical properties of GO [38]. It has been shown that the in-plane properties, i.e. Young's modulus and intrinsic strength, monotonically decrease with increasing degree of oxidation $[39,40]$. Wang et al. have investigated the atomic-scale frictional behavior of GO sheets using density function theory (DFT) including dispersion corrections (DFT-D), and found moderately higher friction forces in GO compared with graphene [41]. More recently, Soler-Crespo et al. have found that the composition of GO also affects the mechanical behavior of GO [42] by using density function-based tight binding (DFTB) technique to systematically characterize the effect of the degree of oxidation and the ratio of functional groups (i.e., epoxide group (-O-) and hydroxyl group (-OH)) on the mechanical performance of GO. They report that epoxide-oxidized GO has relatively larger failure strain than hydroxyl-oxidized GO [42]. Despite tremendous success in understanding and predicting the mechanical behaviors of GO through AA simulations, there are still drawbacks for these computational approaches. The most critical one is that the simulated domain of GO systems is mostly limited to sizes lower than $10 \mathrm{~nm}$, as these approaches are extremely demanding in computational power. This makes it very challenging to assess mechanical behavior of GO based systems at larger scales, limiting our understanding of the behavior of crumpled nanopapers [43], multilayer nanosheets [44], and GO-polymer nanocomposites [21, 23, 45]. Discrete particle methods that avoid artificial homogenization in the mechanical properties of the material are necessary to capture key traits of these systems.

To address these scale-related issues, an upscaling computational technique is required to simulate GO systems beyond current spatiotemporal limitations. Coarse-grained molecular 
dynamics (CG-MD) simulations aimed at predicting the key properties of nanomaterials offer insights into the molecular scale dynamic processes over extended scales and increase computational efficiency dramatically compared to AA simulations [46-49]. Recently, we have presented a novel CG-MD model of graphene achieving $\sim 200$-fold increase in computational speed compared to AA simulations. The model can capture the mechanical properties of multilayer graphene (MLG), including non-linear elasticity, anisotropy at large-deformations and fracture, and also the complex interlayer shear response of graphene [48]. The CG model of MLG is also able to reach experimentally relevant spatio-temporal scales, allowing us to investigate size-dependent mechanical properties of MLG via nanoindentation and tensile deformation simulations $[27,50,51]$. The quantitative agreement between $C G$ simulations of graphene and experiments demonstrates the applicability and predictive power of the CG modeling technique on capturing the mesoscale phenomena as well as explaining physical mechanisms at the molecular level.

Building upon our previous CG framework, here we propose a novel CG model of GO for its mechanical behavior via a bottom-up approach. In this paper, we first provide a description of the CG-MD model of GO and characterize its atomistic properties using DFTB, followed by calibrating the force field parameters using the CG approach. Next, we compare the mechanical properties of GO from both CG-MD and DFTB results. Finally, we demonstrate the applicability of the model by simulating nanoindentation on monolayer GO and measuring the fracture toughness of monolayer GO sheets with different compositions. The CG simulation results agree reasonably well with recent experimental results. 


\section{Methods}

\subsection{CG Model description}

Earlier studies have shown that GO possesses a planar structure with functional groups (mainly epoxide and hydroxyl groups) on both the basal plane and free edges $[5,12,38,52]$. The proposed CG GO model conserves a similar hexagonal lattice structure of graphene with a 4-to-1 mapping scheme for the $s p^{2}$ carbon network. Different from the graphene case, the CG model of GO also includes two other different types of beads, representing hydroxyl- and epoxideoxidized functional regions as illustrated in Fig. 1. While it is extremely difficult to exactly map every GO structure to a CG model, by directly varying the percentages of each type of bead in the CG model, we are able to generate CG GO structures with different degrees of oxidation and compositions, which are key material parameters that govern the mechanical behavior of GO. Note that the degree of oxidation definition in the CG model is defined as the total percentage of both hydroxyl- (type H) and epoxide-oxidized (type E) beads. By also differentiating the bonded interactions between different types of beads, the CG model is able to capture the diversity of mechanical properties of $\mathrm{GO}$ arising from different functional types and degrees of oxidation. 

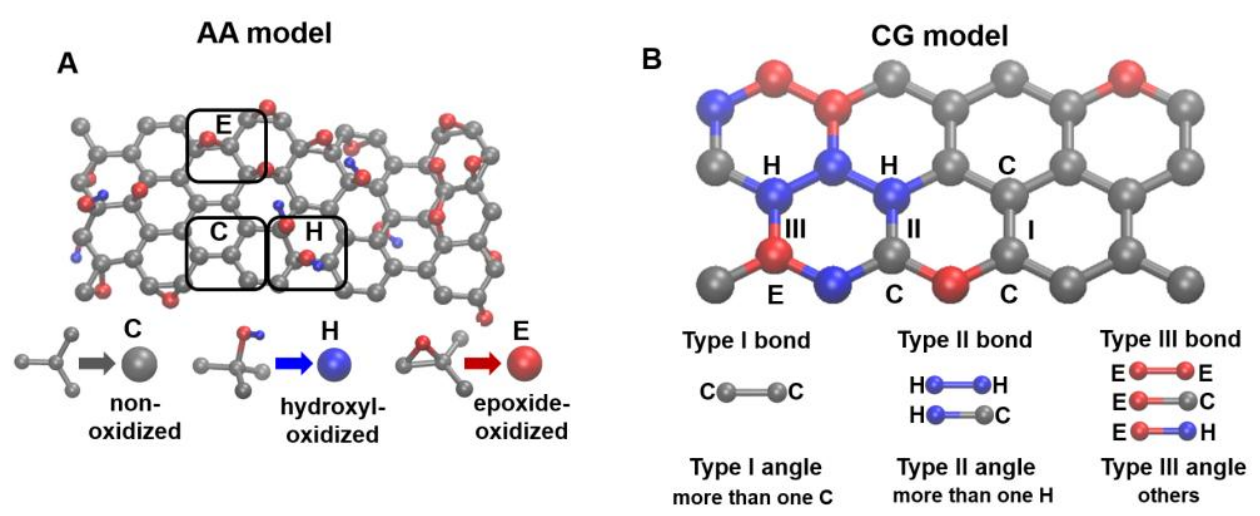

Figure 1. Schematic illustration of coarse-grained (CG) graphene oxide (GO) model. Panel A shows the all-atomistic (AA) GO model and the AA to CG mapping scheme, where three types of CG beads (non-oxidized (C), hydroxyl-oxidized (H) and epoxide-oxidized bead (E)) represent different lattices. Panel B shows the resulting CG structure with different types of CG beads and the classification of bond and angle types. Note that Panel B is not a direct CG representation of the structure in Panel A.

The force field of the CG model includes both bonded (i.e. bond and angle terms) and nonbonded interactions. Thus, the total potential energy of the system can be written as:

$$
E_{\text {pot }}=E_{b}+E_{a}+E_{n b}
$$

where $E_{b}, E_{a}$ and $E_{n b}$ are the sum of the energies of all the bonds, angles and non-bonded interactions of the system, respectively. In this model, we do not include the dihedral term since excluding it simplifies the force field parameterization significantly and increases computational efficiency. Additionally, we have two major considerations to justify this choice. First, we seek to emphasize the in-plane properties for GO (i.e. elastic modulus and uniaxial tensile strength) and interlayer adhesion energies, which are sufficiently captured by the force field given by Eq. (1). Second, the dihedral term only affects the out-of-plane bending stiffness of GO membranes, 
not the properties presented herein. Dihedral terms could be added onto the force field presented here when data on the bending stiffness and its dependence on oxidation becomes available in the future [48].

Accordingly, we define three types of bonds and three types of angles: type I represents the non-oxidized ones, type II represents the hydroxyl-oxidized ones, and type III represents the epoxide-oxidized ones. The classification of bond type is given by the following convention: if and only if both the two beads in a bond are type C, the bond is type I; if there is at least one type $\mathrm{H}$ bead and no type $\mathrm{E}$ bead in the same bond, the bond belongs to type II; otherwise, the bond is type III, as shown in Fig. 1. For angle types: if there is more than one type $\mathrm{C}$ bead in an angle, then the angle is type I, similarly, angle type II is assigned when there is at least one of the involved atoms is type $\mathrm{H}$. All other cases belong to angle type III. The physical basis for the convention is that functional groups affect local regions and a higher amount of functional groups leads to small patches with a bulk response substantially different from pristine graphene. In addition, epoxide groups connect two adjacent carbon atoms, affecting a larger local area; as a result, the type $\mathrm{E}$ bead dominates in the bond and angle classification. In our CG model, the percentage of bonds and angles can simply be changed by prescribing the percentage of different beads. Later we show in the Results section that by using this convention of classification, we are able to capture the dependence of in-plane properties and interlayer adhesion energy of GO sheets on composition and degree of oxidation in good agreement with DFTB calculations.

We use different equilibrium bond lengths $\left(d_{0}\right)$ for the three types of bonds herein, given that functional groups transform pristine $s p^{2}$ bonds to $s p^{3}$ bonds differently. Similar to the CG model of graphene, in which the bond length is double the equilibrium $s p^{2}$ bond length, here we first measure three types of equilibrium bond lengths from a DFTB calculation. We calculate the 
bond length distribution of graphene (Type I bonds), and GO's fully functionalized with hydroxyl and epoxide chemistry (Type II and III bonds, respectively). The average equilibrium bond length in the CG model is taken as double the measured length per our mapping scheme. The equilibrium angle is maintained at $120^{\circ}$, given that for each singular CG case with only one type of bead, the lattice structure maintains the hexagonal symmetry. The functional form of bond type I $\left(V_{b, I}\right)$ is the same as that of the bond in our former CG model for pure graphene, which is represented by the Morse potential form [48]. The reason for using a Morse potential is to capture smooth bond rupture at large strains [53]. For bond types II and III $\left(V_{b, I I} \& I I I\right)$, we choose a piecewise harmonic functional form in order to capture the non-linear behavior of GO. We adopt a harmonic function for all the angle interactions $\left(V_{a}\right)$, and adopt the 6-12 LennardJones (LJ) potential for non-bonded interactions $\left(V_{n b}\right)$. The detailed functional forms are as follows:

$$
\begin{gathered}
V_{b, I}(d)=D_{0}\left[1-e^{-\alpha\left(d-d_{0}\right)}\right]^{2} \\
V_{b, I I \& I I I}(d)= \\
k_{b e}\left(d-d_{0}\right)^{2} \quad d<d_{c 1} \\
\left\{\begin{array}{c}
(2 \mathrm{a}) \\
k_{b p}\left(d-d_{c 2}\right)^{2}+\left[2 k_{b p}\left(d_{c 2}-d_{c 1}\right)+2 k_{b e}\left(d_{c 1}-d_{0}\right)\right]\left(d-d_{c 2}\right)+C_{2} \quad d_{c 2}<d \\
C_{1}=k_{b e}\left(d_{c 1}-d_{0}\right)^{2} \\
C_{2}=k_{b p}\left(d_{c 2}-d_{c 1}\right)^{2}+2 k_{b e}\left(d_{c 1}-d_{0}\right)\left(d_{c 2}-d_{c 1}\right)+C_{1} \\
V_{a}(\theta)=k_{\theta}\left[\theta-\theta_{0}\right]^{2}
\end{array}\right. \\
V_{n b}(r)=4 \varepsilon_{L J}\left[\left(\frac{\sigma_{L J}}{r}\right)^{12}-\left(\frac{\sigma_{L J}}{r}\right)^{6}\right] \quad r<r_{c}
\end{gathered}
$$

Fig. 2(a)(b) show the typical energy and force profiles for bond type II and III, Eq. (2b), respectively. The initial harmonic part in the potential gives rise to linear elastic properties. The change of the slope in the force profile at $d_{c 1}$ results in non-linear behavior in the stress vs. strain 
curve of GO sheets, as shown in Fig. 2(c). Additionally, similar to the Morse potential [53], the potential $V_{b, I I} \& I I I$ can also enable bond rupture, and bond failure strain is only controlled by the critical bond length where the maximum force is achieved (i.e., the bond length $d_{c 2}$ in Fig. 2(b)). Through this approach, the nonlinearity of the stress-strain behavior of GO can be captured phenomenologically. Post-failure plastic behavior and bond reformation events are unlikely to be accurately represented in any coarse-graining approach. Capturing the softening behavior due to plasticity and dynamic bond breaking and reformation accurately requires reactive atomistic force fields that are orders of magnitude slower than CG models.

Finally, for interlayer interactions in AA systems, there are contributions from van der Waals, electrostatic and directional H-bonding interactions. We use a simple LJ potential to account for all interactions as a mean field approach, and in the CG model, all beads interact with other beads within a cutoff distance. Although this choice loses some directional behavior such as $\mathrm{H}$ bonding, the gain in computational efficiency is significant. We note that simple LJ potential is generally used in other force fields of similar degree of coarse-graining CG models, such as the force field of CG model of synthetic polymers and Martini force field for biopolymers[46, 47, 54]. 

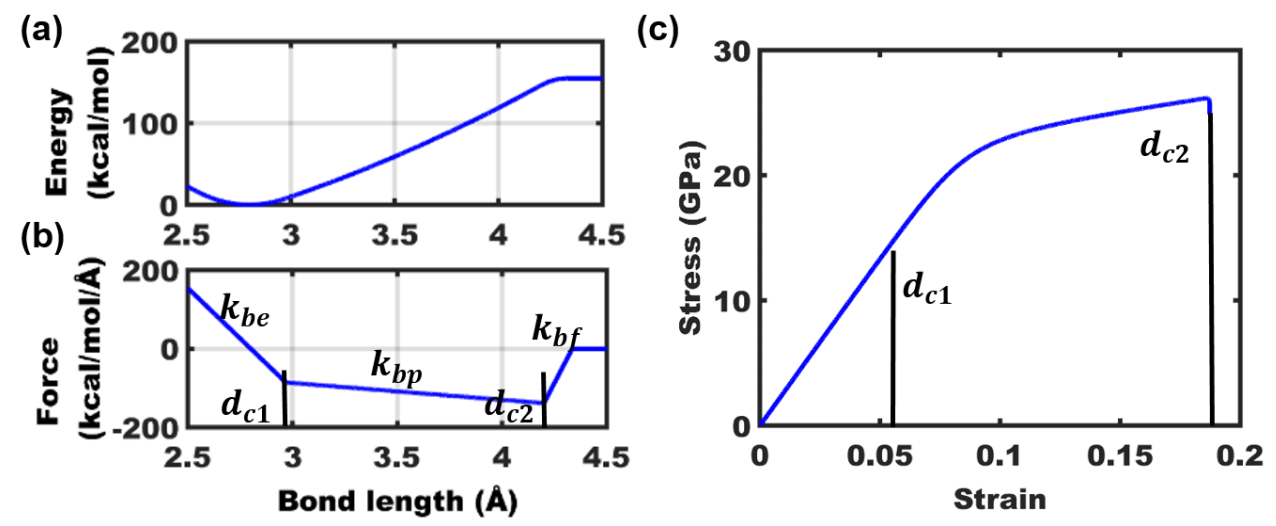

Figure 2. Typical energy (a) and force (b) profiles for bond type II and III potentials. The first kink in the force curve at $d_{c 1}$ induces the nonlinearity in the stress vs. strain curve. The second kink in the force at $d_{c 2}$ results in the failure behavior in the stress vs. strain. (c). Representative stress vs. strain curve of a GO sheet with $60 \%$ degree of oxidation having the epoxide-rich composition using the bond potential shown in (a) (b).

\subsection{Characterization of atomistic GO properties}

The AA simulations are carried out through a series of semi-empirical DFTB calculations using the open-source code CP2K (http://www.cp2k.org/) to determine the mechanical properties of GO as a function of its chemical composition. Through the calculations, we determined the elastic modulus, failure strain under uniaxial tension and interlayer adhesion energy of GO. We first generate AA $20 \times 20 \AA^{2}$ GO monolayer sheets for different degrees of oxidation, based on epoxide or hydroxyl rich compositions. In this study, the term epoxide or hydroxyl rich refers to GO sheets that are only functionalized with epoxide or hydroxyl functional groups respectively. Additionally, GO sheets with a fixed degree of oxidation but variable chemical compositions are generated. A Monte Carlo-based algorithm is employed to determine the favorable locations for functional groups from random choices according to a Boltzmann-like distribution, as discussed 
in previous work and literature $[22,38,42]$. It should be noted that $72 \%$ hydroxyl-rich oxidation and $80 \%$ epoxide-rich oxidation are the maximum oxidation cases that are physically admissible for each functionalization type. Further increasing functional group coverage results in chemical instability of the system during DFTB calculations. Stress-strain curves are determined by assuming the effective monolayer thickness as $\Delta z_{e q}=7.5 \AA$, which is consistent with previous studies $[22,42]$. The Young's moduli $(E)$ are obtained by fitting the material elastic constants in the stress vs. strain curves and using continuum mechanics formulation, as previously shown [42]. The in-plane shear moduli $(S)$ are calculated by using the linear-elastic relationship $S=E / 2(1+v)$, assuming the Poisson's ratio $v$ is a constant equal to 0.16 for all GO sheets, which is taken as the average value for all the degree of oxidation cases according to DFTB calculation results. More details about the computational method and the validation of DFTB calculations are provided in our previous study [42].

To characterize the interlayer adhesion energy, we generate bilayer GO sheets with different composition and degrees of oxidation. The UFF force field is included to account for dispersion effects in the material, as implemented in $\mathrm{CP} 2 \mathrm{~K}$. The adhesion energy can be determined by calculating the total energy of the bilayer system after optimization and subtracting the energy of two separate single sheets. The adhesion energies are calculated for different types of GO bilayer systems, including pure graphene sheets, epoxide-rich GO sheets, hydroxyl-rich GO sheets and GO sheets whose composition results from a combination of both functional groups. We aim to capture the general trends of adhesion energy as the degree of oxidation and composition varies for relatively homogenized systems. While we expected variations across different configurations with the same composition but different distributions, (e.g. localized random 
patches instead of a homogeneous distribution of functional groups), these features are not considered here for calibrating the CG model.

\subsection{Derivation of coarse-grained force field parameters}

Next, we proceed to derive the force field of the CG model by employing the strain energy conservation approach based on all-atomistic (AA) simulations. To calibrate the CG force field parameters, we only choose DFTB results in the armchair direction of three extreme scenarios as references. We use the pure graphene uniaxial tensile results to calibrate bond type I and angle type I parameters, the maximally oxidized hydroxyl-rich composition ( $72 \%$ degree of oxidation) to calibrate bond type II and angle type II parameters, and finally the maximally oxidized epoxide-rich case ( $80 \%$ degree of oxidation) to calibrate bond type III and angle type III parameters. Both results from DFTB calculation and CG model verify that the in-plane properties, i.e., the Young's modulus and uniaxial tensile strength, differ only a little when the degree of oxidation is larger than $70 \%$. This is because in AA simulations the effect of functional groups saturates fairly quickly. The corresponding classification of bond and angle types in the CG model leads to a rapid decrease of type I bonds and angles with increasing percentage of either type $\mathrm{H}$ or $\mathrm{E}$ beads. Thus, the structures at high degrees of oxidation have approximately the same bond and angle compositions. For instance, examining one specific CG structure with $72 \%$ percent of type $\mathrm{H}$ beads reveals that $92 \%$ of the bonds are type II and $81 \%$ of the angles are

type II. This justifies our practice to use these three extreme cases to calibrate the bond and angle parameters. Similarly, we use the bilayer pairwise energies of these extreme cases to calibrate non-bonded interactions. However, we note that the pairwise energy only depends on LJ interactions of beads, so it does not saturate at high degree of oxidation level. Since we use $\sim 80 \%$ degree of oxidation AA structure to calibrate the parameter for $100 \%$ degree of oxidation 
in the CG structure, we need to unify the degree of oxidation concepts by using scaling arguments between AA and CG models to be able to compare the interlayer adhesion energy results. This aspect is discussed further in the Results section.

The necessary mechanical properties to calculate the bond and angle parameters are Young's modulus, in-plane shear modulus, elastic strain and failure strain. LJ parameters are calibrated to match the adhesion energy of bilayer systems. A summary of the target properties is shown in Table 1. 
Table 1. Target Properties for GO

\begin{tabular}{|c|c|c|c|c|c|}
\hline & $\begin{array}{c}\text { Young's } \\
\text { modulus }(\boldsymbol{E}) \\
\mathrm{GPa}\end{array}$ & $\begin{array}{c}\text { In-plane shear } \\
\text { modulus }(\boldsymbol{S}) \\
\mathrm{GPa}\end{array}$ & $\begin{array}{c}\text { Elastic } \\
\text { strain } \\
\left(\boldsymbol{\varepsilon}_{\text {ela }}\right)\end{array}$ & $\begin{array}{c}\text { Failure } \\
\text { strain } \\
\left(\boldsymbol{\varepsilon}_{\boldsymbol{m a x}}\right)\end{array}$ & $\begin{array}{c}\text { Adhesion } \\
\text { energy } \\
\mathbf{m J} / \mathbf{m}^{\mathbf{2}}\end{array}$ \\
\hline Graphene & 530 & 228 & - & $21 \%$ & 31 \\
\hline $\begin{array}{c}\text { Hydroxyl- } \\
\text { oxidized GO }\end{array}$ & 285 & 121 & $8 \%$ & $10 \%$ & 156 \\
\hline $\begin{array}{c}\text { Epoxide- } \\
\text { oxidized GO }\end{array}$ & 230 & 99 & $8 \%$ & $25 \%$ & 97 \\
\hline
\end{tabular}

* The elastic modulus is calculated by assuming the effective thickness of the monolayer GO as $7.5 \AA$ [42].

After having established target properties, we proceed to describe the calibration strategy. Since the Morse potential (bond type I) can be approximated by a harmonic function $V_{b, I}(d)=$ $D_{0}\left[1-e^{-\alpha\left(d-d_{0}\right)}\right]^{2} \approx k_{b e}\left(d-d_{0}\right)^{2}$, where $k_{b e}=D_{0} \alpha^{2}$, all bond types can be approximated as harmonic in the small deformation regime. Gillis has derived the relationship between the force constants of the harmonic bonds and angles of the hexagonal lattice and the elastic constants of the total sheet [55], which we have utilized in our previous CG model of graphene [48]:

$$
\begin{gathered}
k_{b}=\frac{\sqrt{3} c E S}{2(4 S-E)} \\
k_{\theta}=\frac{\sqrt{3} d_{0}^{2} c E S}{12(3 E-4 S)}
\end{gathered}
$$

where $c=2 \Delta z_{e q}$, and $\Delta z_{e q}=7.5 \AA$. Thus, all parameters involved in the angle potentials and $k_{b e}$ of bond potentials can be calculated directly. It should be noted that the parameters calculated using Eq. (5) and (6) do not depend on the value of $\Delta z_{e q}$ as long as it is kept the same as the assumed effective monolayer thickness for DFTB calculation, since $k_{b}$ and $k_{\theta}$ scales linearly with the $2 \mathrm{D}$ modulus, $\Delta z_{e q} E$. 
For the Morse bond potential (i.e. bond type I) in MD simulation, a bond typically breaks at the elongation where the force reaches a maximum, or equivalently the inflection point in the energy profile [53]. The parameter $\alpha$ is directly related to this inflection point in the energy profile. We set the failure criterion of the bonds according to the failure strain of GO sheets, as the same in the CG model of graphene[48]. Thus, we can calculate the parameter $\alpha$ as $\alpha=$ $\log 2 /\left(\varepsilon_{\max } d_{0}\right)$, where $\varepsilon_{\max }$ is the failure strain as obtained from DFTB results. Once $k_{b e}$ and $\alpha$ have been determined, the parameter $D_{0}$ can be calculated using the relation $D_{0}=k_{b e} / \alpha^{2}$. For bond types II and III, the parameter $d_{c 1}$ linearly scales with the linear elastic limit strain $\varepsilon_{e l a}$. We conduct uniaxial tensile simulation using the CG model with different values of $d_{c 1}$ and pick a value that conserves $\varepsilon_{\text {ela }}$ of DFTB results. The slope of the second linear part is related to nonlinearity of the stress-strain curve in the region before failure. The average slope of the postelastic region observed from DFTB calculations is conserved by choosing a corresponding $k_{b p}$ value. Similar to the Morse bond potential, the $d_{c 2}$ parameter is directly related to failure strain, and is calibrated to match the failure strain from DFTB calculations. Our CG results also suggest that the slope parameter $k_{b f}$ does not affect the failure strain and stress vs. strain curve, and thus we specify it as $k_{b f}=-2 k_{b e}$. During simulations, we delete bonds once they are stretched to $d_{c u t}$, which is slightly larger than $d_{c 2}$. Thus, all the parameters of the bond and angle potentials can be determined.

The only term left for the force-field that requires calibration is the non-bonded interaction, which is important for capturing the properties of multilayer GO assemblies. The parameter $\varepsilon$ represents the depth of the potential well and directly relates to the adhesion energy, and the parameter $\sigma$ controls the interlayer spacing. In this study, we use constant interlayer spacing $7.5 \AA$ for different scenarios given that the interlayer spacing increases immediately with the 
presence of functional groups and it differs less than $10 \%$ at high functional group density according to DFTB results. The constant interlayer spacing also simplifies our model and results in an identical $\sigma_{L J}$ value of $7.48 \AA$ for all bead types. An identical cutoff value of $20 \AA$ is chosen to balance between including as much portion of the attractive well as needed and without experiencing too much computational slowdown. Specifically, the adhesion energy calculated using cutoff value of $20 \AA$ is in less than $5 \%$ difference with that calculated using cutoff value of $40 \AA$. By testing different values of $\varepsilon$ for a system with only one type of bead at low temperature (i.e. negligible entropic effects), we find that $\varepsilon$ linearly scales with the non-bonded interaction energy of bilayer systems. We calibrate the parameter $\varepsilon$ for three types of beads according to the DFTB results of the three scenarios in Table 1 at fixed interlayer spacing $7.5 \AA$. The pair coefficient for interactions between different types of beads is obtained via a Lorentz-Berthelot mixing rule: $\sigma_{I J}=\frac{\sigma_{I I}+\sigma_{J J}}{2}, \varepsilon_{I J}=\sqrt{\varepsilon_{I I} \varepsilon_{J J}}$.

A summary of all the CG force-field functional forms and the corresponding calibrated parameters is presented in Table 2. 
Table 2. Functional forms and calibrated parameters of the CG model force-field

\begin{tabular}{|c|c|c|}
\hline Interaction & Functional form & Parameters \\
\hline \multirow{3}{*}{ Bond } & Type I: $\begin{aligned} V_{b, I}(d) & =D_{0}\left[1-e^{-\alpha\left(d-d_{0}\right)}\right]^{2} \\
d_{0} & =2.86 \AA\end{aligned}$ & $\begin{array}{c}D_{0}=443.07 \frac{\mathrm{kcal}}{\mathrm{mol}} \\
\alpha=1.154 \\
d_{c \mathrm{ut}}=3.7 \AA\end{array}$ \\
\hline & \multirow{2}{*}{$\begin{array}{c}\text { Type II \& III: } \\
V_{b, I I \& I I I}(d)= \\
k_{b e}\left(d-d_{0}\right)^{2} \quad d<d_{c 1} \\
\left\{\begin{array}{cc}k_{b p}\left(d-d_{c 1}\right)^{2}+2 k_{b e}\left(d_{c 1}-d_{0}\right)\left(d-d_{c 1}\right)+C_{1} \quad d_{c 1}<d<d_{c 2} \\
k_{b f}\left(d-d_{c 2}\right)^{2}+\left[2 k_{b p}\left(d_{c 2}-d_{c 1}\right)+2 k_{b e}\left(d_{c 1}-d_{0}\right)\right]\left(d-d_{c 2}\right)+C_{2} \quad d_{c 2}<d \\
C_{1}=k_{b e}\left(d_{c 1}-d_{0}\right)^{2} \\
C_{2}=k_{b p}\left(d_{c 2}-d_{c 1}\right)^{2}+2 k_{b e}\left(d_{c 1}-d_{0}\right)\left(d_{c 2}-d_{c 1}\right)+C_{1}\end{array}\right.\end{array}$} & $\begin{array}{c}\text { Type II: } \\
d_{0}=2.94 \AA \\
d_{c 1}=3.12 \AA \\
d_{c 2}=3.46 \AA \\
d_{c \mathrm{ut}}=3.5 \AA \\
k_{b e}=317.34 \frac{\mathrm{kcal}}{\mathrm{mol} \AA^{2}} \\
k_{b p}=126.94 \frac{\mathrm{kcal}}{\mathrm{mol} \AA^{2}} \\
k_{b f}=634.68 \frac{\mathrm{kcal}}{\mathrm{mol}^{2}}\end{array}$ \\
\hline & & $\begin{array}{c}\text { Type III: } \\
d_{0}=2.80 \AA \\
d_{c 1}=3.00 \AA \\
d_{c 2}=4.20 \AA \\
d_{\text {cut }}=4.3 \AA \\
k_{b e}=256.1 \frac{\mathrm{kcal}}{\mathrm{mol} \AA^{2}} \\
k_{b p}=21.34 \frac{\mathrm{kcal}}{\mathrm{mol} \AA^{2}} \\
k_{b f}=512.2 \frac{\mathrm{kcal}}{\mathrm{mol} \AA^{2}}\end{array}$ \\
\hline \multirow{3}{*}{ Angle } & \multirow{3}{*}{$\begin{array}{c}V_{a}(\theta)=k_{\theta}\left[\theta-\theta_{0}\right]^{2} \\
\theta_{0}=120^{\circ}\end{array}$} & Type I: $k_{\theta}=456.61 \frac{\mathrm{kcal}}{\mathrm{mol}}$ \\
\hline & & Type II: $k_{\theta}=259.47 \frac{\mathrm{kcal}}{\mathrm{mol}}$ \\
\hline & & Type III: $k_{\theta}=189.93 \frac{\mathrm{kcal}}{\mathrm{mol}}$ \\
\hline \multirow{3}{*}{$\begin{array}{l}\text { Non- } \\
\text { bonded }\end{array}$} & \multirow{3}{*}{$\begin{array}{c}V_{n b}(r)=4 \varepsilon_{L J}\left[\left(\frac{\sigma_{L J}}{r}\right)^{12}-\left(\frac{\sigma_{L J}}{r}\right)^{6}\right] \\
\sigma_{L J}=7.48 \AA\end{array}$} & $\begin{array}{c}\text { Type C: } \\
\varepsilon_{L J}=0.0255 \frac{\mathrm{kcal}}{\mathrm{mol}}\end{array}$ \\
\hline & & $\begin{array}{c}\text { Type H: } \\
\varepsilon_{L J}=0.128 \frac{\mathrm{kcal}}{\mathrm{mol}}\end{array}$ \\
\hline & & $\begin{array}{c}\text { Type E: } \\
\varepsilon_{L J}=0.0797 \frac{\mathrm{kcal}}{\mathrm{mol}}\end{array}$ \\
\hline
\end{tabular}




\subsection{Protocols for the CG-MD simulations}

The molecular dynamics code LAMMPS [56] is used to conduct all the CG-MD simulations. We choose the same time-step of $4 \mathrm{fs}$ as that used in the CG model of graphene [48]. To simulate the in-plane mechanical response, we use a monolayer sheet of dimensions $\sim 50 \times 50 \mathrm{~nm}^{2}$. The system contains $\sim 24000$ beads in total and periodic boundary conditions (PBCs) are applied in all the directions, while a $10 \mathrm{~nm}$ empty space exists on either side of the sheet in the out-of-plane direction. This appropriate model size is determined by running multiple simulations with increasing size until convergence is achieved in the stress-strain results. We find that for smaller systems, the failure strain and maximum stress are relatively higher, and this size effect might arise from the specific classification of bonds and angles in the CG model. The systems are first minimized and equilibrated in a NPT assemble at $T=300 \mathrm{~K}$ and zero pressure in the in-plane directions. After equilibration, the strain-controlled uniaxial tensile test is performed by deforming the simulation box at a constant strain rate of $1 \times 10^{8} S^{-1}$ in the armchair direction. During deformation, we constrained the out of plane displacements of all the beads in order to maintain the uniaxial tensile condition and minimize entropic elastic behavior [57], given that there is negligible entropic elastic behavior in DFTB calculations on uniaxial tension of small sheets. Because of the large box size in the dimension normal to the sheet, the stress computed using the virial theorem for the box does not correspond to the actual stress in the sheet, and needs to be corrected by multiplying with the ratio of the box dimension to the actual monolayer sheet thickness. We use the same effective monolayer thickness $7.5 \AA$ used in DFTB calculations for this purpose. We calibrate the parameters at $300 \mathrm{~K}$ since DFTB calculations have been shown to match experimental results at room temperature[42]. Moreover, our CG model is anticipated to be applied more often to study room temperature scenarios. However, we note that the 
Young's modulus is not sensitive to the temperature, and by decreasing the temperature to $10 \mathrm{~K}$, the Young's modulus only increases less than $2 \%$. The strength shows a decrease with increasing temperature, and the difference is up to $20 \%$ between $10 \mathrm{~K}$ and $300 \mathrm{~K}$ for all the cases presented herein. The failure strain of the CG model is mainly governed by the bond elongation, and higher temperature increases the probability of the bonds to reach cutoff bond length. As a result, higher temperature leads to earlier failure of the sheet. The dependency of Young's modulus and strength on temperature is similar to an earlier study on pristine graphene using the adaptive intermolecular reactive empirical bond order (AIREBO) potential $[58,59]$.

The bilayer adhesion simulations involve two GO sheets parallel to each other with dimensions $\sim 20 \times 20 \mathrm{~nm}^{2}$. PBCs are applied in the in-plane dimensions, and again there is a $10 \mathrm{~nm}$ empty space on either side of the sheets in the out-of-plane direction. We use a relatively large system to get a better statistical sampling. We also test different sizes of systems ranging from $8 \times$ $8 \mathrm{~nm}^{2}$ to $40 \times 40 \mathrm{~nm}^{2}$, and the variation of the adhesion energy calculated is within $5 \%$. After initial minimization, the system is equilibrated in the NVT ensemble at temperature $T=300 \mathrm{~K}$. After equilibration, the energy due to the interlayer pair-wise non-bonded interactions is measured with the intralayer non-bonded interactions being turned off. The adhesion energy at $10 \mathrm{~K}$ is also calculated, and it is found to be less than 5\% larger than the adhesion energy at room temperature, thus verifying that the entropic effect on the adhesion energy is negligible.

To test the predictive capabilities of the developed CG model, we perform nanoindentation simulations of monolayer and multilayer GO sheets. We use a similar size as our previous study on pristine graphene (indenter radius, $R=4 \mathrm{~nm}$, and membrane radius, $a=25 \mathrm{~nm}$ ) for the simulation [50]. Although the size selected is still 20 times smaller than those used in recent experiments [22], previous studies show that this size is enough to illustrate mesoscale 
mechanisms by using size-scaling analysis [48]. The circular indentation region is in the center of a square sheet with length $\sim 60 \mathrm{~nm}$ in order to resemble the experimental setting that a square sheet is suspended over a circular hole. Nonperiodic boundary conditions are employed due to the finite nature of the system. The simulation is also conducted at $300 \mathrm{~K}$. We simulate the indenter as a rigid sphere that interacts with GO beads via a repulsive harmonic force with a force constant set to be $1000 \mathrm{kcal} / \mathrm{mol} \cdot \AA^{3}$. The indenter is moved with a constant downwards velocity of $5 \mathrm{~m} / \mathrm{s}$ from the initial position where it has no interaction with the GO beads. During the indentation process, the beads outside of the circular region are fixed by a stiff harmonic spring with spring constant $10000 \mathrm{kcal} / \mathrm{mol} \cdot \AA^{3}$ and the beads within the circular region are set freestanding without any constraints. In our previous study, explicit sensitivity analysis confirmed that the indentation rate has no observable effect on the mechanical response of CG system [50].

Finally, we apply our CG model to study the fracture toughness of different GO sheets with different compositions. The same dimension size as the uniaxial tension case is used. Central cracks with different lengths are generated by deleting the beads and corresponding bond and angle interactions. The cracks are aligned with the zigzag direction and tensile loading is applied in the armchair direction. An equivalent deformation procedure to that used in uniaxial tensile deformation is applied.

\section{Results and discussion}

Having derived all of the force field parameters for the CG models based on the three extreme cases, we validate the CG model by testing it for different degrees of oxidation and compositions. In Fig. 3, we compare CG and DFTB uniaxial tensile results in the armchair direction for GO sheets with different degrees of oxidation on hydroxyl-rich compositions. We 
note that the CG model shows anisotropy in the uniaxial tensile results of armchair and zigzag direction since we adopt a hexagonal symmetric lattice, although DFTB results do not show such an effect due to breakage of symmetry. Specifically, the failure strain and failure stress in the zigzag direction are generally $\sim 15 \%$ larger than those in the armchair direction, and our previous results of graphene show similar anisotropy in both directions[48]. Nonetheless, the general decreasing trend of Young's modulus and strength with increasing degree of oxidation is well captured for both directions in the CG model. In addition, the brittle failure of the hydroxyloxidation case is captured by using the piecewise potential form for the new bond type II.

By calculating the elastic moduli and strength for different degrees of oxidation, DFTB and CG results show that Young's modulus decreases faster at lower degrees of oxidation than that at higher degrees of oxidation, as shown in Fig. 4. In other words, a small amount of functional group coverage results in an obvious deterioration of both Young's modulus and strength due to disruption of the $s p^{2}$ network of graphene, while the impact of further oxidation becomes diminished at higher percentages of hydroxylation. The Young's modulus for each case is within $10 \%$ error compared with the DFTB results. It is a remarkable accomplishment of the model that the parameterization for the $72 \%$ case alone is sufficient to capture the uniaxial tensile behaviors of other degrees of oxidation cases with reasonable accuracy. The strength deviation for lower degrees of oxidation cases is relatively larger than the Young's modulus case. Specifically, the deviations are $23.1 \%, 17.5 \%, 4.6 \%$ and $14.5 \%$ for the four oxidation cases listed in Fig. 3. The relatively large deviation for low degree of oxidation is due to the fact that failure strains are almost a constant for atomistic GO sheets in DFTB calculations, while in CG models, for low degree of oxidation cases, type II bonds, with smaller force constants, are more significantly stretched than type I bonds, and this localized behavior results in early failures of GO sheets with 
lower degree of oxidation in CG simulations. It is foreseeable that more localized behavior would be present in larger systems. 
(a)

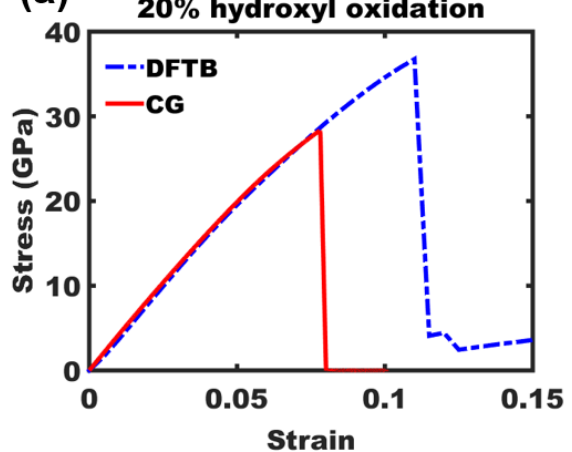

(c)

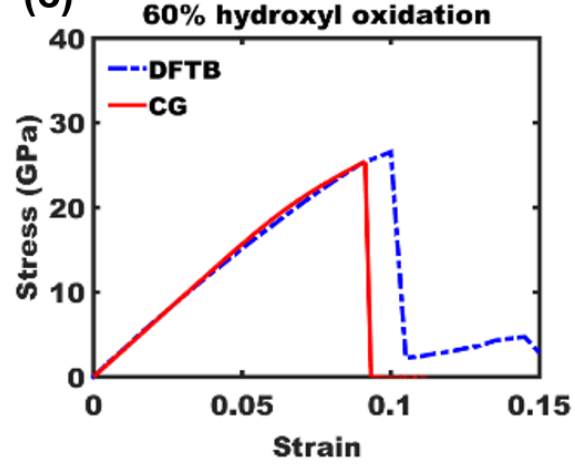

(b)

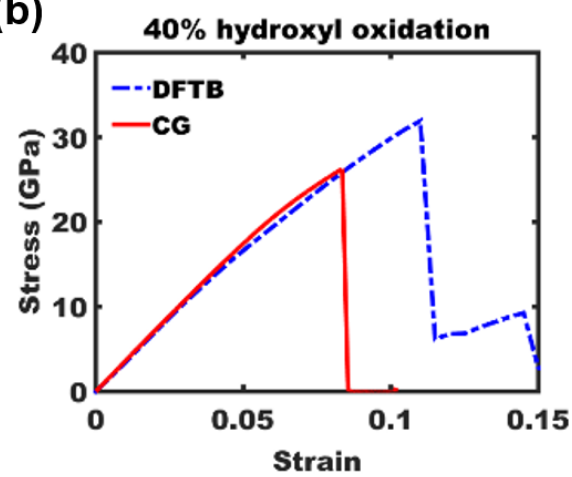

(d)

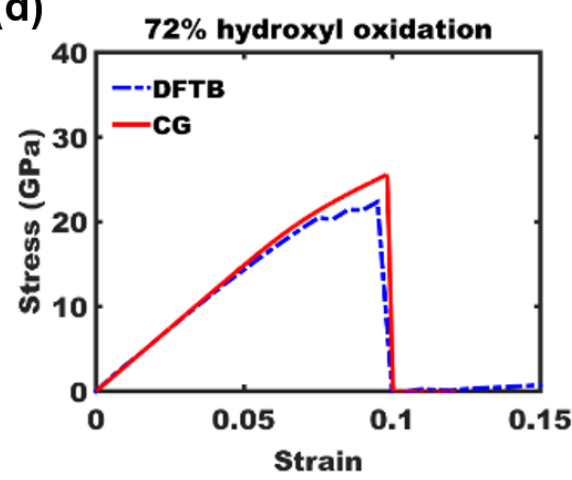

Figure 3. Uniaxial stress vs. strain results (armchair direction) from CG simulations and DFTB calculations for GO sheets with hydroxyl-rich compositions at different degrees of oxidation.
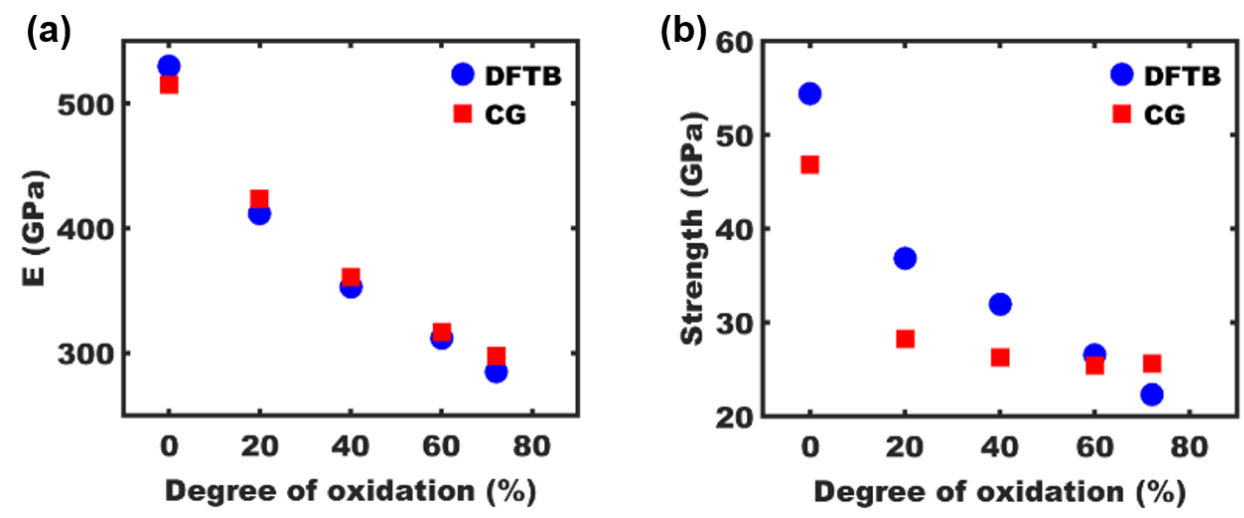

Figure 4. Comparison of the elastic moduli (a) and the tensile strengths (b) between CG model and DFTB calculation for GO sheets with hydroxyl-rich compositions at different degrees of oxidation. 
Similarly, Fig. 5 shows the comparison between CG uniaxial tensile results of epoxide-rich CG and DFTB calculations. Generally speaking, GO sheets with a high epoxide composition exhibit a relative larger failure strain and ductility, which is defined as the difference between failure strain and elastic strain. The higher ductility of epoxide-rich cases can be attributed to an epoxide-to-ether transformation at the atomic level [22]. For the CG model performance, the Young's modulus and strength captured and their decreasing trend agree closely with DFTB results, as shown in Fig. 6 . In addition, since the parameter of bond type III has a larger $d_{c 2}$, the CG model is able to reproduce the large failure strain and ductility for the epoxide-oxidation case, and it also reproduces the general increase of failure strain with increasing epoxide coverage. It should be noted that the CG model shows less variability for different initial configurations than AA models. More specifically, the Young's moduli have negligible differences with different random distributions of the beads, and the standard deviation for strength is only $1 \%$ of the averaged value. This is because the CG model treats the complicated chemical microstructure with a simple strain based criteria and a simpler lattice structure, resulting in a CG model that simplifies and stabilizes mechanical behavior during deformation. 
(a)

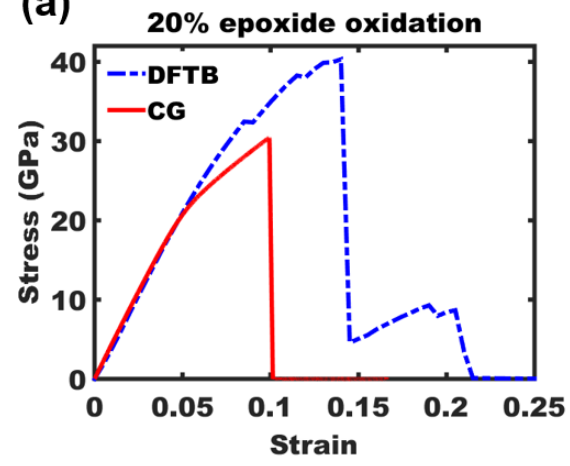

(c) $60 \%$ epoxide oxidation

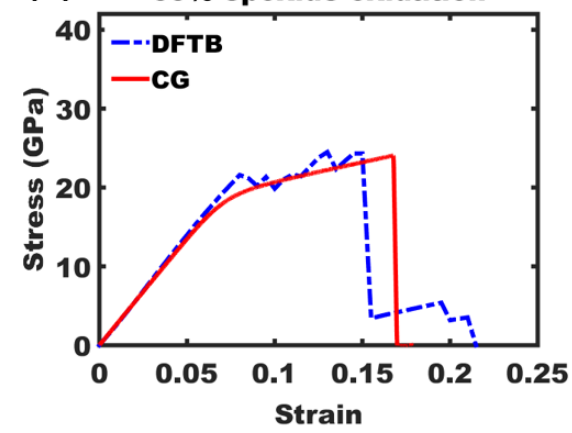

(b)

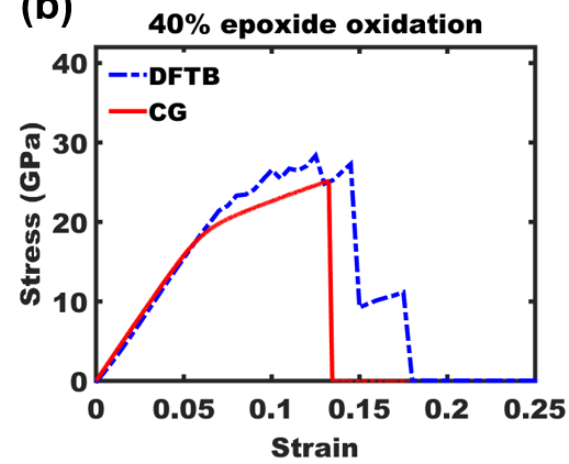

(d) $80 \%$ epoxide oxidation

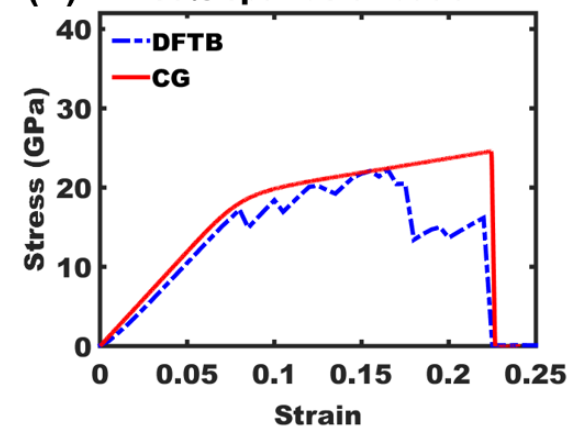

Figure 5. Uniaxial stress vs. strain results (armchair direction) from CG simulation and DFTB calculation for GO sheets with epoxide-rich compositions at different degrees of oxidation.
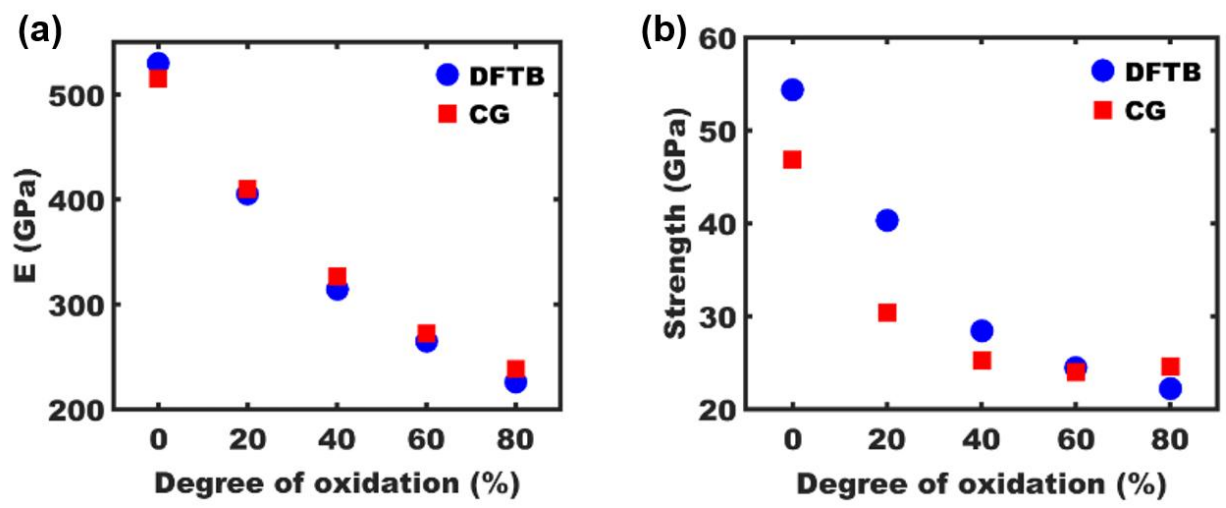

Figure 6. Comparison of the elastic moduli (a) and the tensile strengths (b) between CG model and DFTB calculation for GO sheets with epoxide-rich compositions at different degrees of oxidation. 
Having verified the CG model for both hydroxyl-rich and epoxide-rich compositions, we then test the CG model for a wider set of compositions that include combinations of epoxide and hydroxyl functionalization. First, we define the ratio of epoxide and hydroxyl functional groups as:

$$
\delta=\frac{N_{\text {epoxides }}}{N_{\text {epoxides }}+N_{\text {hydroxyls }}}(7)
$$

where $N_{\text {epoxides }}$ and $N_{\text {hydroxyls }}$ are the total number of epoxide and hydroxyl groups in DFTB calculations, while they represent the total number of type $\mathrm{E}$ and type $\mathrm{H}$ beads in $\mathrm{CG}$ simulations. DFTB results indicate that for a given degree of oxidation ( $70 \%$ in this case), brittle to ductile failure behavior can be observed by increasing the epoxide group percentage, which also leads to an appreciable enhancement in toughness, which is defined as the area below the stress vs. strain curve [42]. As shown in Fig. 7, the CG model is able to quantitatively reproduce the increasing toughness trend with increasing epoxide-oxidized beads percentage for combined functionalization cases. Fig. 7(c) shows the comparison of ductility, which is defined as the difference between linear elastic limit strain $\varepsilon_{\text {ela }}$ and failure strain $\varepsilon_{\max }$, between DFTB results and our CG model, and the agreement is remarkable. These results demonstrate the predictive capabilities of our CG model given that the model is only calibrated based on the three extreme cases. 

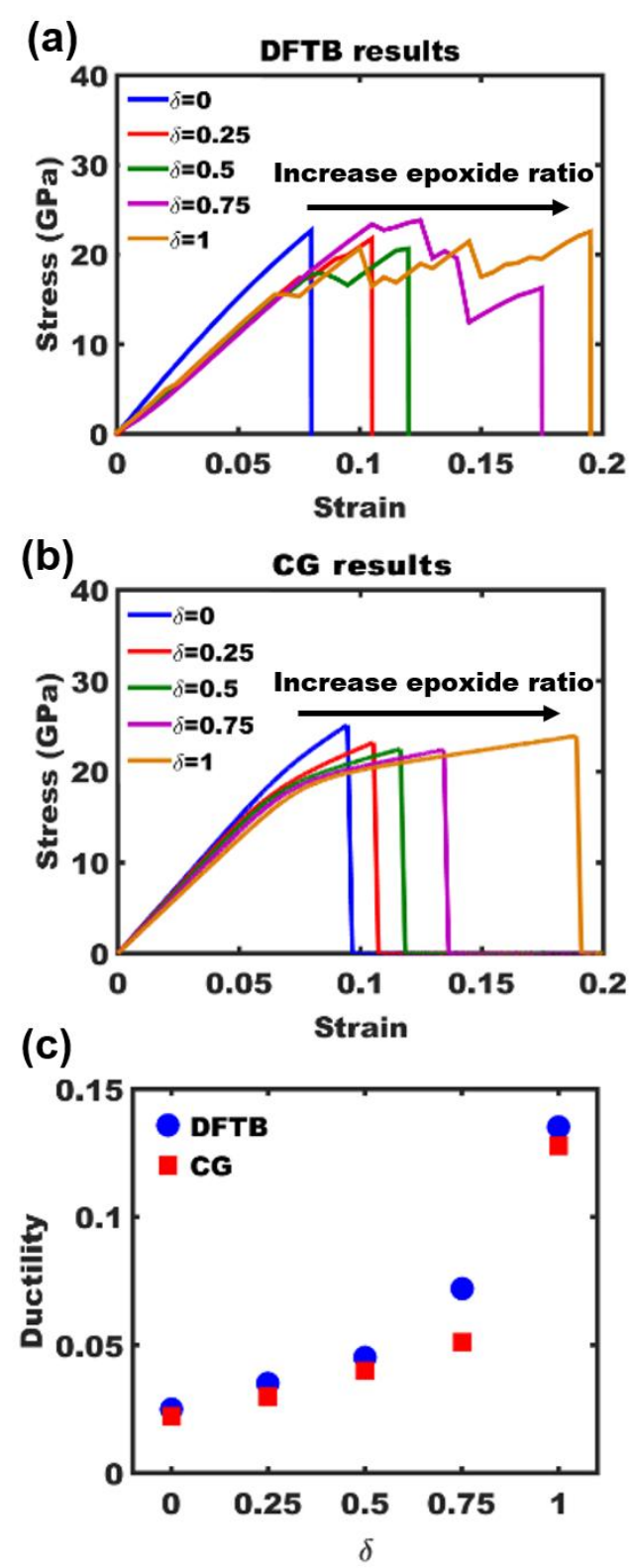

Figure 7. In-plane mechanical behavior of the DFTB results (a) and the CG results (b) for different hydroxyl/epoxide ratio at $70 \%$ degree of oxidation. In (a), $\delta$ is defined as the ratio between the number of epoxide groups and the total number of epoxide and hydroxyl groups, and in (b), $\delta$ is defined as the ratio between the number of type $\mathrm{E}$ beads and the total number of type $\mathrm{H}$ and type $\mathrm{E}$ beads. (c). Ductility vs. hydroxyl/epoxide ratio $\delta$ for both DFTB (circle) and CG (square) results. 
For the interlayer interaction performance, Fig. 8 shows the interlayer adhesion energy difference for three functionalization cases predicted from the CG model: hydroxyl-rich, epoxide-rich and a combined composition with a 1:1 ratio between hydroxyl and epoxide functional groups. By differentiating the interactions for the three types of beads, our CG model can quantitatively match the relationship between adhesion energy and degree of oxidation, and also capture the interlayer adhesion difference resulted from different functionalization. Specifically, the CG model captures the increasing adhesion energy with increasing degree of oxidation, as well as the higher adhesion energies of hydroxyl-rich cases. The resulting adhesion energies of combined compositions lie in between the two functionalization cases. We also run four atomistic cases of combined compositions in DFTB: 10\%/10\% hydroxyl/epoxide, 20\%/20\% hydroxyl/epoxide, $30 \% / 30 \%$ hydroxyl/epoxide and $40 \% / 40 \%$ hydroxyl/epoxide. The DFTB results also show increasing adhesion energy with increasing degree of oxidation. We note that since we calibrate the LJ parameters according to $\sim 80 \%$ degree of oxidation cases, we must convert the degree of oxidation of all atomistic cases by multiplying 1.25 so that the degree of oxidation is comparable between DFTB calculation and CG model results, for example, a $40 \% / 40 \%$ hydroxyl/epoxide atomistic case corresponds to a $50 \% / 50 \%$ hydroxyl/epoxide CG case. After the conversion of degree of oxidation, the DFTB results all lie near the CG predictions as shown in Fig. 8, and this consistency further shows the predictive capability of the CG model on the interlayer interactions. 


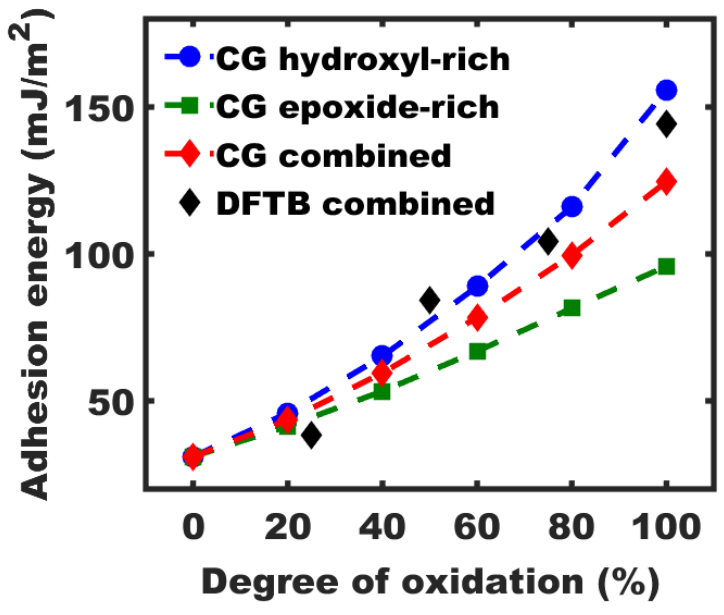

Figure 8. Comparison between the predicted adhesion energies for three functionalization cases: hydroxyl-oxidation only, epoxide-oxidation only and combined oxidation with 1:1 ratio between hydroxyl and epoxide. DFTB adhesion energy results for combined oxidation with 1:1 ratio after conversion of the degree of oxidation are shown in black solid diamonds.

The model has been shown to quantitatively capture both the in-plane and interlayer mechanical responses of GO sheets with different compositions and degrees of oxidation. We then apply the CG model to simulate monolayer GO nanoindentation behavior, in which the GO sheet undergoes large deformation until failure. Nanoindentation is one of the most widely used experimental techniques to investigate the mechanical properties of $2 \mathrm{D}$ materials $[50,51]$. Although during the nanoindentation, there is out-of-plane deformation involved, it has been verified that the in-plane biaxial tension still governs the total deformation. Specifically, by testing the CG model of pristine graphene with and without dihedral terms[48], and the difference in the force-deflection curve is negligible, and even at maximum deflection, the dihedral energy contribution is less than $1 \%$ of the bond energy. As a result, even without the 
dihedral terms, the $\mathrm{CG}$ model is adequate to capture the realistic nanoindentation mechanical response.

In our simulation, we use a $4 \mathrm{~nm}$ radius spherical punch to indent on $25 \mathrm{~nm}$ radius circular regions of three types of GO sheets: one without functional groups, as in a pristine graphene sheet, one with a 70\% oxidized hydroxyl-rich GO sheet and the last case is a $70 \%$ oxidized epoxide-rich GO sheet. We note that it is unrealistic to simulate such systems using DFTB technique due to prohibitive computational costs, while the same computation takes less than 2 hours of wall-clock time in 16 processors using our CG model. We use a theoretical model to fit the load-displacement curve from the simulations [51], in which the force vs. deflection behavior can be approximated using the equation:

$$
F=\sigma_{0} \pi t \delta+\frac{E t}{q^{3} a^{2}} \delta^{3}
$$

where $F$ is the applied force, $\sigma_{0}$ is the pretension in the membrane, $\delta$ is the central deflection or indentation depth, $a$ is the film radius, $q=1.02$ is a dimensionless constant derived from the Poisson's ratio of the system, $t=\Delta z_{e q}$ is the effective thickness of the membrane. We use the Young's modulus $E$ calculated from the uniaxial tensile test for each type of GO sheet to fit the curves, and also $\sigma_{0}=0$, given that the sheets are fully relaxed before indentation loading in the simulations.

Fig. 9 illustrates that the analytical fits deviate from simulation data for large deformation in the graphene and epoxide oxidation cases. The non-linear elastic behavior of graphene at large deformations corresponds to the deviation from the linear elastic assumption of the theoretical model. For epoxide oxidized cases, the deviations after reaching an indentation depth of $5 \mathrm{~nm}$ is due to the non-linear ductility of epoxide oxidized GO. However, for hydroxyl oxidized case, 
since the sheet is linear elastic until brittle failure as shown in the uniaxial tensile results, there should not be any significant deviation from the analytical fit, which is corroborated by the data shown in Fig. 9. These findings are significant because force vs. deflection responses for GO membranes reported experimentally have been classified into two classes, corresponding to ductile and brittle failure modes $[22,60]$. Our CG results suggests that ductile failure occurs when the indenter contact area is predominantly occupied by epoxide groups, while brittle failure is observed when the indenter interacts with a hydroxyl-rich area, or an area that has high defect density. Our findings illustrate the importance of explicit representation of these discrete heterogeneity regions in the CG model to explain the experimental data.

We then derive the nominal strength from the peak load in the force-displacement curve using equation $\sigma_{\text {max }}=\sqrt{F E /(4 \pi R t)}$ (where $F$ is the rupture force, $R$ is the indenter radius, and $t$ is the film thickness). We note that this equation is for a linear elastic circular membrane, and thus it would overestimate the strengths of GO sheets due to material nonlinearity. Specifically, the derived nominal strengths for the three cases in Fig. 9 are 55.3 GPa, 28.5 GPa, 35.3 GPa for graphene sheet, $70 \%$ hydroxyl-rich GO sheet and 70\% epoxide-rich GO sheet, respectively. The measured strengths for the same materials in uniaxial tests are around $49 \mathrm{GPa}, 26 \mathrm{GPa}$ and 25 $\mathrm{GPa}$, respectively, taken as the average strength in both armchair and zigzag directions. The results indicate that with the highest nonlinearity for $70 \%$ epoxide-rich GO sheets, the nominal strength derived from indentation test has the most overestimation when compared to its actual strength. In our previous study [50], we have shown that the effective strength extracted from indentation measurement is affected by the interlayer shear strength for multilayer systems. This study ascertains that for a monolayer system, the measurement of strength via indentation could be affected by in-plane nonlinearity and heterogeneity, which should be considered in future 
strength measurements of 2D materials where better statistical sampling may be needed to obtain accurate material properties.

(a)

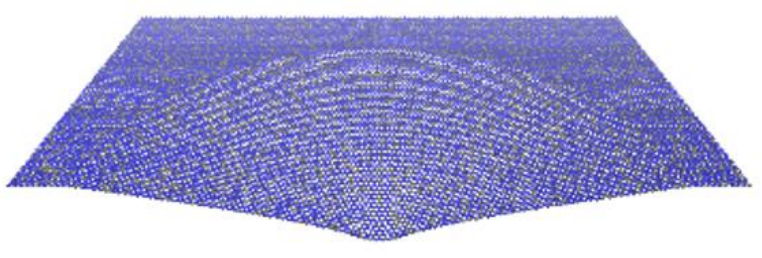

(b)

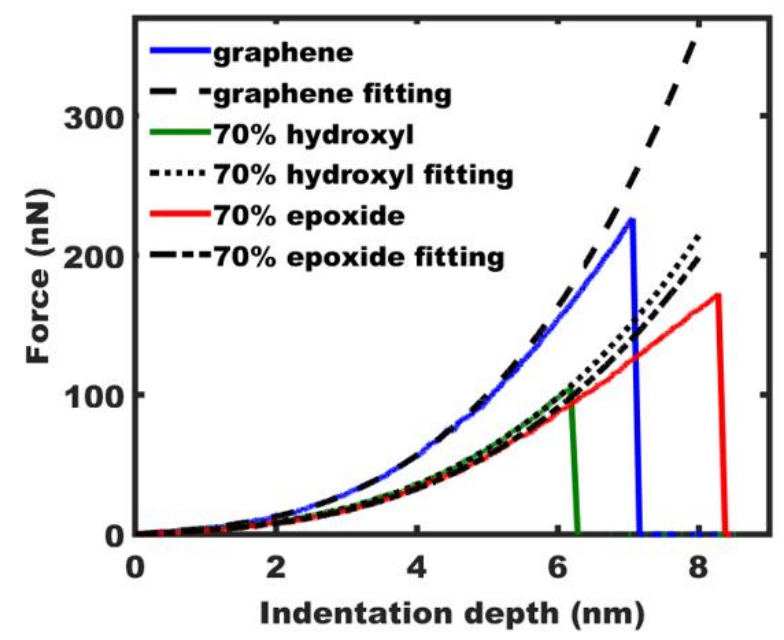

Figure 9. (a). Snapshot of a 70\% hydroxyl-rich GO sheet during indentation (we only show half of the sheet for clarity). (b). Nanoindentation simulation results of CG GO sheets (solid line) with theory fitting (dashed line) for three cases: pure graphene, 70\% hydroxyl oxidation GO and $70 \%$ epoxide oxidation GO, respectively.

It has been proposed that classic Griffith theory of brittle fracture may be applicable to graphene and GO [44, 61]. Unlike ductile and quasi-brittle materials, such as metals and polymeric materials, which are difficult to simulate fracture in atomistic simulations due to the large size of plastic zone or process zone[62,63], classic brittle fracture has been successfully 
studied with atomistic simulations[61, 64]. As further validation of our model, here we measure the critical stress intensity factor $K_{c}$, which determines the linear-elastic fracture toughness of the material, for the same three types of GO sheets studied during nanoindentation using this developed CG model.

According to the Griffith fracture criterion for a central crack of length $2 a_{0}$ :

$$
\sigma_{c} \sqrt{\pi a_{0}}=\sqrt{2 \gamma E}
$$

where the left-hand side is the critical stress intensity factor $K_{c}=\sigma_{c} \sqrt{\pi a_{0}}$, and the right-hand side term depends only on material properties ( $E$ is the Young's modulus and $\gamma$ is the surface energy, i.e., edge energy for 2D material like graphene).

To measure $K_{c}$, the failure far-field stress is measured for specimens with different central crack length. Fig. 10(a) shows the configuration of the simulation system, and after reaching critical stress $\sigma_{c}$, the crack propagates catastrophically through the whole sheet, as captured by Fig. 10(b). Brittle fracture is also manifested by the linear response in the stress-strain curves, as shown in Fig. 10(c). 

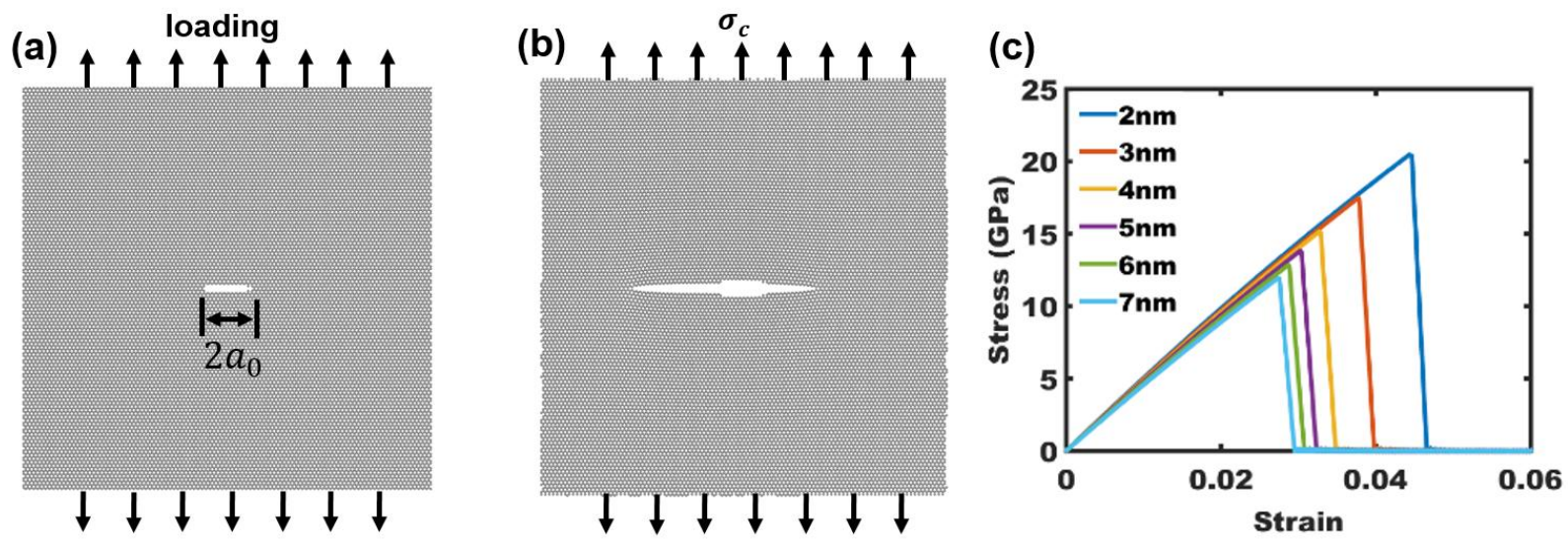

Figure 10. (a) Schematic of the CG pristine graphene sheet with a central crack, $a_{0}=3 \mathrm{~nm}$ in this case. (b). The state of catastrophic crack propagation. (c). Stress-strain relationships for different crack lengths.

Table 3. Simulation data of crack size, critical fracture stress and critical stress intensity factor $K_{c}$.

\begin{tabular}{|c|c|c|}
\hline$a_{0}(\mathrm{~nm})$ & $\sigma_{c}(G P a)$ & $K_{c}(M P a \sqrt{m})$ \\
\hline 2 & 20.5 & 1.63 \\
\hline 3 & 17.5 & 1.70 \\
\hline 4 & 15.2 & 1.70 \\
\hline 5 & 13.8 & 1.73 \\
\hline 6 & 12.8 & 1.76 \\
\hline 7 & 11.9 & 1.76 \\
\hline Average & & 1.71 \\
\hline Standard Deviation & & 0.05 \\
\hline
\end{tabular}

Table 3 lists the results of critical fracture stress and critical stress intensity factor for different specimens. Our results show that $K_{c}$ is approximately constant, and for large crack lengths, $K_{c}$ shows even less variance, further corroborating that Eq. (9) is ideal for long and sharp cracks. It 
should be noted that $K_{c}$ values in Table 3 are calculated by assuming a monolayer thickness of $0.75 \mathrm{~nm}$. By converting to a monolayer thickness of $0.34 \mathrm{~nm}$ such as that of graphene, $K_{c}=$ 3.77 MPa $\sqrt{m}$ which is consistent to other MD studies and experimentally measured values of $4.0 \pm 0.6 \mathrm{MPa} \sqrt{m}[61,64]$. Since we also capture the Young's modulus of graphene accurately, according to Eq. (9), the edge energy is also conserved in our CG model, and this validates our strain energy conservation approach.

Then, the same procedure is adopted to measure the fracture toughness of both hydroxyl-rich and epoxide-rich GO sheets with a 70\% degree of oxidation. Interestingly, although epoxide GO shows an obvious non-linear response during uniaxial tension, it exhibits nearly linear response before failure and smaller failure strain when a defect is present, as seen in brittle fracture of catastrophic crack propagation (Fig. 11(b)), indicating the likely applicability of Griffith theory. The measured $K_{c}$ is $1.00 \pm 0.04 M P a \sqrt{m}$ for the hydroxyl case, and $1.16 \pm 0.03 \mathrm{MPa} \sqrt{\mathrm{m}}$ for the epoxide case. $K_{c}$ values of GO are generally lower than that of graphene, and this is because the fracture toughness of brittle materials is mainly governed by bond stiffness and strength, or the Young's modulus in a general sense, as indicated by the right-hand side of Eq. (10). Notably, the $K_{c}$ of epoxide-rich GO is $16 \%$ larger than that of hydroxyl oxidized GO. These results further corroborate the influence of chemistry on the fracture toughness and in agreement with previous reports that epoxide-rich GO has higher toughness than hydroxyl-rich GO [42].

We note with interest that the fracture toughness of materials can be influenced by crack blunting, crack orientation and polycrystalline microstructure, as investigated in recent studies for graphene case [57, 61]. In addition, for GO sheets, the fracture properties will likely be further modified by crack tip chemistry and the distribution of different functionalized regions. These issues should provide fruitful topics for future studies. 

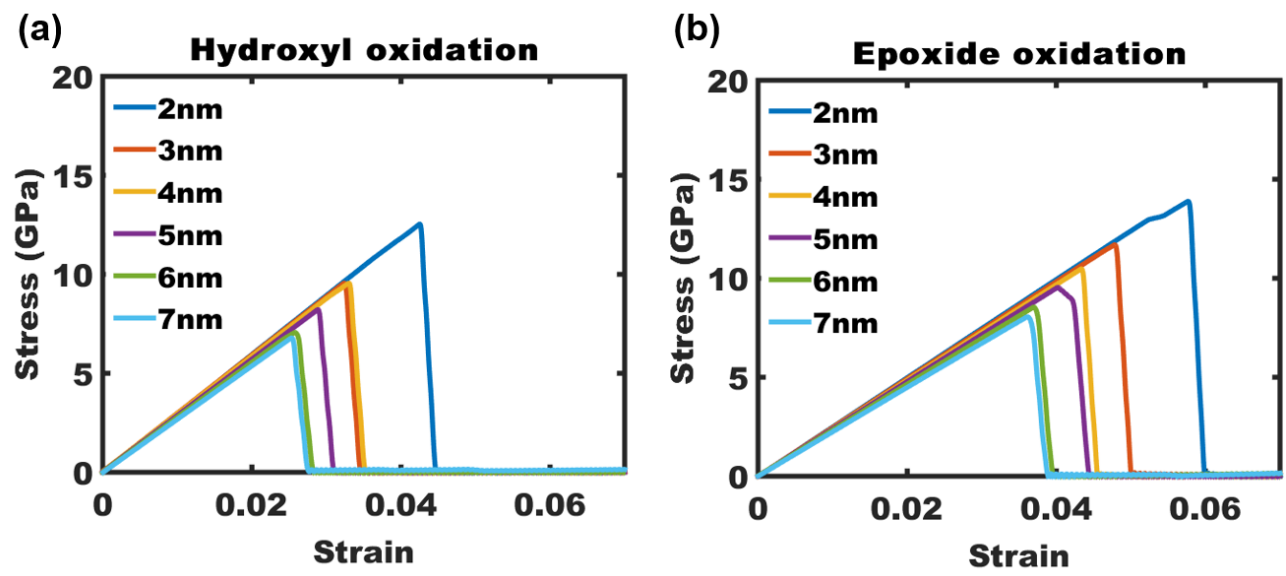

Figure 11. Stress-strain relationships for different crack lengths of hydroxyl-rich GO (a) and epoxide-rich GO (b).

\section{Conclusion}

In this work, we present a CG-MD model of GO which is capable of reproducing the deteriorating mechanical properties of GO with increasing degree of oxidation (functional group density) while improving the interfacial adhesion energy. The model captures different properties that result from different functionalization types (i.e. compositions) with at least $\sim 8000$-fold increase in computational speed compared to DFTB calculations of equivalent size. We use a strain energy conservation approach to calibrate the potential parameters according to DFTB calculations. Despite the simplicity of the force-field and the fact that the model is only calibrated according to three extreme cases: pure graphene case, maximally oxidized hydroxylrich case and maximally oxidized epoxide-rich case, we show that our model can qualitatively capture the Young's modulus, uniaxial tensile strength and interlayer adhesion energy of any degree of oxidation and various ratios of epoxide to hydroxyl group functionalization cases. After validating the model, we simulate the mechanical response of monolayer GO under 
nanoindentation, and we show that either ductile or brittle failure occurs depending on different contact area chemistry, consistent with previous experimental observations. We also apply the CG model to measure the fracture toughness of different GO sheets, and the results suggest the applicability of classic Griffith theory of brittle fracture. We measured the highest fracture toughness for graphene, and the fracture toughness value is consistent with previous experimental and computational studies. We also observe that epoxide-rich GO is tougher than hydroxyl-rich GO due to extensible epoxide bonds. These analyses illustrate the capability of the CG model to capture mesoscale large-deformation and failure mechanisms reasonably well. Thus, our CG model offers a great platform to study the influence of crack shape, crack orientation and polycrystalline microstructure on fracture properties.

Additionally, our model is compatible with existing models of peptides and polymers in terms of the degree of coarse-graining [46,47], which makes it suitable for studying the interfacial and mechanical properties of GO-based nanocomposites. The model can be readily used to implement additional features such as defects and islands of functional groups as seen experimentally for GO, instead of randomly distributed functional groups. Our work sets the stage for future studies on the role of heterogeneity on the mechanical properties of multilayer GO sheets and has the potential to lend key insights into failure mechanisms pertaining to GObased nanocomposites. 


\section{Acknowledgements}

The authors acknowledge funding by the National Science Foundation (DMREF award CMMI1235480). HDE also acknowledges the support of ARO through MURI Award No. W911NF-081-0541. The authors acknowledge the support from the Departments of Civil and Environmental Engineering and Mechanical Engineering at Northwestern University, as well as the Northwestern University High Performance Computing Center for a supercomputing grant. DFT and DFTB calculations were carried out on the TACC Stampede high performance computing facility, at the University of Texas at Austin, through the support of NSF XSEDE Award Nos. TG-MSS140028 and TG-MSS150003. R.A.S-C. acknowledges support from NSF through the Graduate Research Fellowships Program (GRFP) and partial support from the Northwestern University Ryan Fellowship \& International Institute for Nanotechnology. W.X. acknowledges the support from the NIST-CHiMaD Postdoctoral Fellowship. The authors also thank S.P. Nathamgari and M.R. Roenbeck for helpful discussions. 


\section{References}

[1] A.K. Geim, K.S. Novoselov, The rise of graphene, Nature materials 6(3) (2007) 183-191.

[2] K.S. Novoselov, A.K. Geim, S.V. Morozov, D. Jiang, Y. Zhang, S.V. Dubonos, I.V. Grigorieva, A.A. Firsov, Electric Field Effect in Atomically Thin Carbon Films, Science 306 (2004).

[3] X. Li, Y. Zhu, W. Cai, M. Borysiak, B. Han, D. Chen, R.D. Piner, L. Colombo, R.S. Ruoff, Transfer of largearea graphene films for high-performance transparent conductive electrodes, Nano letters 9(12) (2009) 4359-4363.

[4] W. Cai, Y. Zhu, X. Li, R.D. Piner, R.S. Ruoff, Large area few-layer graphene/graphite films as transparent thin conducting electrodes, Applied Physics Letters 95(12) (2009) 123115.

[5] Y. Zhu, S. Murali, W. Cai, X. Li, J.W. Suk, J.R. Potts, R.S. Ruoff, Graphene and Graphene Oxide Synthesis Properties and Applications, Advanced Materials (2010).

[6] R.J. Young, I.A. Kinloch, L. Gong, K.S. Novoselov, The mechanics of graphene nanocomposites: a review, Composites Science and Technology 72(12) (2012) 1459-1476.

[7] I.-S. Byun, D. Yoon, J.S. Choi, I. Hwang, D.H. Lee, M.J. Lee, T. Kawai, Y.-W. Son, Q. Jia, H. Cheong, Nanoscale lithography on monolayer graphene using hydrogenation and oxidation, ACS nano 5(8) (2011) 6417-6424.

[8] P. Liu, Y. Zhang, A theoretical analysis of frictional and defect characteristics of graphene probed by a capped single-walled carbon nanotube, Carbon 49(11) (2011) 3687-3697.

[9] L. Gong, I.A. Kinloch, R.J. Young, I. Riaz, R. Jalil, K.S. Novoselov, Interfacial stress transfer in a graphene monolayer nanocomposite, Advanced Materials 22(24) (2010) 2694-2697.

[10] L. Gong, R.J. Young, I.A. Kinloch, I. Riaz, R. Jalil, K.S. Novoselov, Optimizing the reinforcement of polymer-based nanocomposites by graphene, ACS nano 6(3) (2012) 2086-2095.

[11] D.A. Dikin, S. Stankovich, E.J. Zimney, R.D. Piner, G.H.B. Dommett, G. Evmenenko, S.T. Nguyen, R.S. Ruoff, Preparation and characterization of graphene oxide paper, Nature 448 (2007).

[12] D.R. Dreyer, S. Park, C.W. Bielawski, R.S. Ruoff, The chemistry of graphene oxide, Chemical Society Reviews 39(1) (2010) 228-240.

[13] H. Kim, A.A. Abdala, C.W. Macosko, Graphene/polymer nanocomposites, Macromolecules 43(16)

(2010) 6515-6530.

[14] G. Titelman, V. Gelman, S. Bron, R. Khalfin, Y. Cohen, H. Bianco-Peled, Characteristics and microstructure of aqueous colloidal dispersions of graphite oxide, Carbon 43(3) (2005) 641-649.

[15] S. Stankovich, R.D. Piner, X. Chen, N. Wu, S.T. Nguyen, R.S. Ruoff, Stable aqueous dispersions of graphitic nanoplatelets via the reduction of exfoliated graphite oxide in the presence of poly (sodium 4styrenesulfonate), Journal of Materials Chemistry 16(2) (2006) 155-158.

[16] S. Stankovich, D.A. Dikin, R.D. Piner, K.A. Kohlhaas, A. Kleinhammes, Y. Jia, Y. Wu, S.T. Nguyen, R.S. Ruoff, Synthesis of graphene-based nanosheets via chemical reduction of exfoliated graphite oxide, Carbon 45(7) (2007) 1558-1565.

[17] Y. Gao, L.-Q. Liu, S.-Z. Zu, K. Peng, D. Zhou, B.-H. Han, Z. Zhang, The effect of interlayer adhesion on the mechanical behaviors of macroscopic graphene oxide papers, ACS nano 5(3) (2011) 2134-2141.

[18] N.V. Medhekar, A. Ramasubramaniam, R.S. Ruoff, V.B. Shenoy, Hydrogen bond networks in graphene oxide composite paper: structure and mechanical properties, Acs Nano 4(4) (2010) 2300-2306. [19] O.C. Compton, S.W. Cranford, K.W. Putz, Z. An, L.C. Brinson, M.J. Buehler, S.T. Nguyen, Tuning the mechanical properties of graphene oxide paper and its associated polymer nanocomposites by controlling cooperative intersheet hydrogen bonding, ACS nano 6(3) (2012) 2008-2019.

[20] D. Cai, M. Song, A simple route to enhance the interface between graphite oxide nanoplatelets and a semi-crystalline polymer for stress transfer, Nanotechnology 20(31) (2009) 315708. 
[21] J. Liang, Y. Huang, L. Zhang, Y. Wang, Y. Ma, T. Guo, Y. Chen, Molecular-Level Dispersion of Graphene into Poly(vinyl alcohol) and Effective Reinforcement of their Nanocomposites, Advanced Functional Materials 19 (2009).

[22] X. Wei, L. Mao, R.A. Soler-Crespo, J.T. Paci, J. Huang, S.T. Nguyen, H.D. Espinosa, Plasticity and ductility in graphene oxide through a mechanochemically induced damage tolerance mechanism, Nature communications 6 (2015).

[23] Y. Xu, W. Hong, H. Bai, C. Li, G. Shi, Strong and ductile poly (vinyl alcohol)/graphene oxide composite films with a layered structure, Carbon 47(15) (2009) 3538-3543.

[24] S. Stankovich, D.A. Dikin, G.H. Dommett, K.M. Kohlhaas, E.J. Zimney, E.A. Stach, R.D. Piner, S.T. Nguyen, R.S. Ruoff, Graphene-based composite materials, nature 442(7100) (2006) 282-286.

[25] T. Ramanathan, A.A. Abdala, S. Stankovich, D.A. Dikin, M. Herrera-Alonso, R.D. Piner, D.H. Adamson, H.C. Schniepp, X. Chen, R.S. Ruoff, S.T. Nguyen, I.A. Aksay, R.K. Prud'Homme, L.C. Brinson, Functionalized graphene sheets for polymer nanocomposites, Nat. Nanotechnol. 3(6) (2008) 327-331.

[26] J.R. Potts, D.R. Dreyer, C.W. Bielawski, R.S. Ruoff, Graphene-based polymer nanocomposites, Polymer 52(1) (2011) 5-25.

[27] W. Xia, L. Ruiz, N.M. Pugno, S. Keten, Critical length scales and strain localization govern the mechanical performance of multi-layer graphene assemblies, Nanoscale 8(12) (2016) 6456-6462.

[28] W. Xia, J. Song, Z. Meng, C. Shao, S. Keten, Designing multi-layer graphene-based assemblies for enhanced toughness in nacre-inspired nanocomposites, Molecular Systems Design \& Engineering (2016). [29] C. Shao, S. Keten, Stiffness Enhancement in Nacre-Inspired Nanocomposites due to Nanoconfinement, Sci. Rep. 5 (2015) 16452.

[30] Y. Matsuo, K. Tahara, Y. Sugie, Structure and thermal properties of poly (ethylene oxide)intercalated graphite oxide, Carbon 35(1) (1997) 113-120.

[31] P. Liu, K. Gong, P. Xiao, M. Xiao, Preparation and characterization of poly (vinyl acetate)-intercalated graphite oxide nanocomposite, Journal of Materials Chemistry 10(4) (2000) 933-935.

[32] J. Xu, Y. Hu, L. Song, Q. Wang, W. Fan, G. Liao, Z. Chen, Thermal analysis of poly (vinyl alcohol)/graphite oxide intercalated composites, Polymer degradation and stability 73(1) (2001) 29-31.

[33] W. Kai, Y. Hirota, L. Hua, Y. Inoue, Thermal and mechanical properties of a poly

( $\epsilon$-caprolactone)/graphite oxide composite, Journal of Applied Polymer Science 107(3) (2008) 1395-1400.

[34] N.A. Kotov, I. Dékány, J.H. Fendler, Ultrathin graphite oxide-polyelectrolyte composites prepared by self-assembly: Transition between conductive and non-conductive states, Advanced Materials 8(8) (1996) 637-641.

[35] T. Cassagneau, J.H. Fendler, High density rechargeable lithium-ion batteries self-assembled from graphite oxide nanoplatelets and polyelectrolytes, Advanced Materials 10(11) (1998) 877-881.

[36] N.I. Kovtyukhova, P.J. Ollivier, B.R. Martin, T.E. Mallouk, S.A. Chizhik, E.V. Buzaneva, A.D. Gorchinskiy, Layer-by-layer assembly of ultrathin composite films from micron-sized graphite oxide sheets and polycations, Chemistry of Materials 11(3) (1999) 771-778.

[37] J. Wu, Q. Tang, H. Sun, J. Lin, H. Ao, M. Huang, Y. Huang, Conducting film from graphite oxide nanoplatelets and poly (acrylic acid) by layer-by-layer self-assembly, Langmuir 24(9) (2008) 4800-4805.

[38] J.T. Paci, T. Belytschko, G.C. Schatz, Computational studies of the structure, behavior upon heating, and mechanical properties of graphite oxide, The Journal of Physical Chemistry C 111(49) (2007) 1809918111.

[39] J.Z. Lizhao Liu, Jijun Zhao and Feng Liu, Mechanical properties of graphene oxides, Nanoscale 4 (2012).

[40] Q. Zheng, Y. Geng, S. Wang, Z. Li, J.-K. Kim, Effects of functional groups on the mechanical and wrinkling properties of graphene sheets, Carbon 48(15) (2010) 4315-4322.

[41] L.-F. Wang, T.-B. Ma, Y.-Z. Hu, H. Wang, Atomic-scale friction in graphene oxide: an interfacial interaction perspective from first-principles calculations, Physical Review B 86(12) (2012) 125436. 
[42] R.A. Soler-Crespo, W. Gao, P. Xiao, X. Wei, J.T. Paci, G. Henkelman, H.D. Espinosa, Engineering the Mechanical Properties of Monolayer Graphene Oxide at the Atomic Level, The Journal of Physical Chemistry Letters (2016).

[43] X. Ma, M.R. Zachariah, C.D. Zangmeister, Crumpled nanopaper from graphene oxide, Nano letters 12(1) (2011) 486-489.

[44] C. Cao, M. Daly, B. Chen, J.Y. Howe, C.V. Singh, T. Filleter, Y. Sun, Strengthening in graphene oxide nanosheets: Bridging the gap between interplanar and intraplanar fracture, Nano letters 15(10) (2015) 6528-6534.

[45] A.M. Beese, Z. An, S. Sarkar, S.S.P. Nathamgari, H.D. Espinosa, S.T. Nguyen, Defect-Tolerant Nanocomposites through Bio-Inspired Stiffness Modulation, Adv. Funct. Mater. 24(19) (2014) 2883-2891. [46] D.D. Hsu, W. Xia, S.G. Arturo, S. Keten, Thermomechanically consistent and temperature transferable coarse-graining of atactic polystyrene, Macromolecules 48(9) (2015) 3057-3068. [47] D.D. Hsu, W. Xia, S.G. Arturo, S. Keten, Systematic method for thermomechanically consistent coarse-graining: a universal model for methacrylate-based polymers, Journal of chemical theory and computation 10(6) (2014) 2514-2527.

[48] L. Ruiz, W. Xia, Z. Meng, S. Keten, A coarse-grained model for the mechanical behavior of multilayer graphene, Carbon 82 (2014) 103-115.

[49] S. Cranford, D. Sen, M.J. Buehler, Meso-origami: folding multilayer graphene sheets, Applied physics letters 95(12) (2009) 123121.

[50] X. Wei, Z. Meng, L. Ruiz, W. Xia, C. Lee, J.W. Kysar, J.C. Hone, S. Keten, H.D. Espinosa, Recoverable Slippage Mechanism in Multilayer Graphene Leads to Repeatable Energy Dissipation, ACS nano (2016). [51] C. Lee, X. Wei, J.W. Kysar, J. Hone, Measurement of the elastic properties and intrinsic strength of monolayer graphene, Science 321(5887) (2008) 385-388.

[52] A. Lerf, H. He, M. Forster, J. Klinowski, Structure of graphite oxide revisited, The Journal of Physical Chemistry B 102(23) (1998) 4477-4482.

[53] T. Belytschko, S. Xiao, G. Schatz, R. Ruoff, Atomistic simulations of nanotube fracture, Physical Review B 65(23) (2002) 235430.

[54] S.J. Marrink, H.J. Risselada, S. Yefimov, D.P. Tieleman, A.H. De Vries, The MARTINI force field: coarse grained model for biomolecular simulations, The journal of physical chemistry B 111(27) (2007) 7812-

7824.

[55] P.P. Gills, Calculating the elastic constants of graphite, Carbon 22 (1984).

[56] S. Plimpton, Fast Parallel Algorithms for Short-Range Molecular-Dynamics, J. Comput. Phys. 117(1) (1995) 1-19.

[57] T. Zhang, X. Li, S. Kadkhodaei, H. Gao, Flaw insensitive fracture in nanocrystalline graphene, Nano letters 12(9) (2012) 4605-4610.

[58] H. Zhao, N. Aluru, Temperature and strain-rate dependent fracture strength of graphene, Journal of Applied Physics 108(6) (2010) 064321.

[59] S.J. Stuart, A.B. Tutein, J.A. Harrison, A reactive potential for hydrocarbons with intermolecular interactions, The Journal of chemical physics 112(14) (2000) 6472-6486.

[60] C. Cao, M. Daly, C.V. Singh, Y. Sun, T. Filleter, High strength measurement of monolayer graphene oxide, Carbon 81 (2015) 497-504.

[61] P. Zhang, L. Ma, F. Fan, Z. Zeng, C. Peng, P.E. Loya, Z. Liu, Y. Gong, J. Zhang, X. Zhang, Fracture toughness of graphene, Nature communications 5 (2014).

[62] J. Rottler, S. Barsky, M.O. Robbins, Cracks and crazes: on calculating the macroscopic fracture energy of glassy polymers from molecular simulations, Phys. Rev. Lett. 89(14) (2002) 148304.

[63] Z. Meng, M.A. Bessa, W. Xia, W. Kam Liu, S. Keten, Predicting the Macroscopic Fracture Energy of Epoxy Resins from Atomistic Molecular Simulations, Macromolecules (2016).

[64] M.J. Moura, M. Marder, Tearing of free-standing graphene, Physical Review E 88(3) (2013) 032405. 
DFTB calculation on graphene oxide
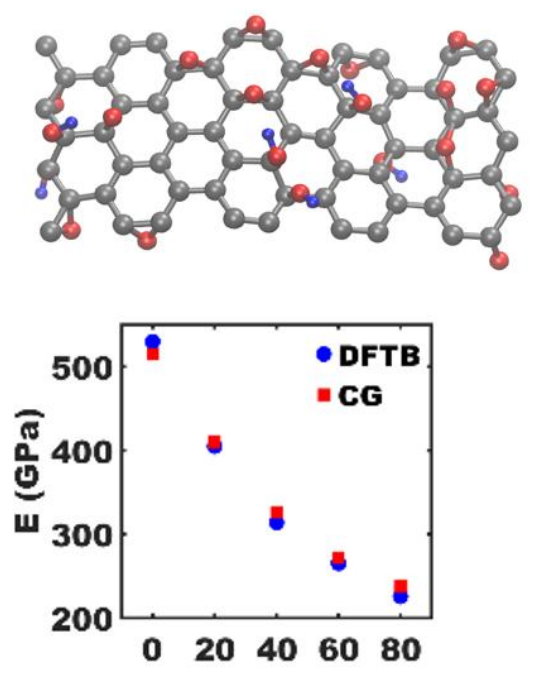

Degree of oxidation (\%)

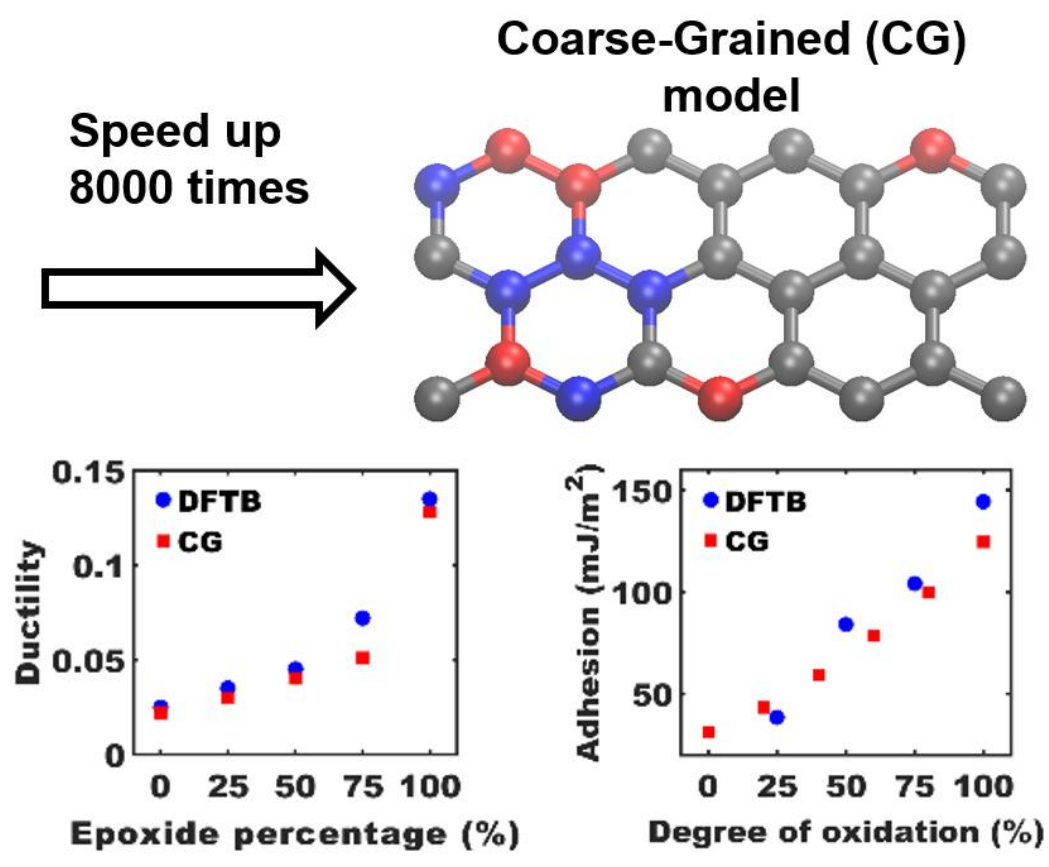

